FACULDADE DE CIÊNCIAS ECONô MICAS dA UFRGS
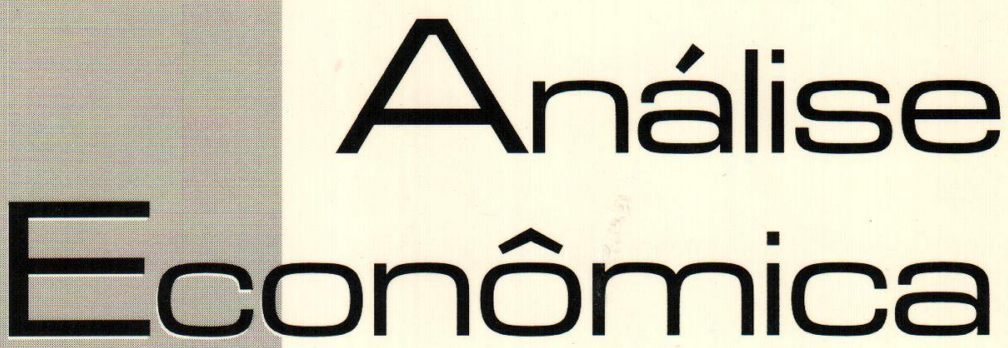

ESCOLHA DE PORTFÓLIO, INVESTIMENTO E NĀO. NEUTRALIDADE DA MOEDA

JOSÉ LUIS OREIRO

REFORMAS NA AROUITETURA FINANCEIRA INTERNACIONAL: NOVIDADES NO FRONT? ANDRÉ MOREIRA CUNHA

MERCOSUR'S CHANGE IN TRADE PATTERNS ANDRÉ FILIPE ZAGO DE AZEVEDO

O IMPACTO DA COMPOSICÃO SETORIAL, DOS FLUXOS INTRA-SETORIAIS E DA ABERTURA COMERCIAL NA PARTICIPAÇÁO DE MERCADO DAS EXPORTAÇÓES BRASILEIRAS

CLÉSIO LOURENÇO XAVIER E EMERSON FERNANDES MARÇAL

O EFEITO BALASSA-SAMUELSON E A PARIDADE DO PODER DE COMPRA NA ECONOMIA BRASILEIRA CLÁUDIO ROBERTO FÓFFANO VASCONCELOS

CICLOS Y FLUCTUACIONES FINANCIERAS: LA IRREGULAR DINÁMICA ECONÓMICA

SARY LEVY-CARCIENTE

O PENSAMENTO DE KARL POPPER: AS DIFERENTES INTERPRETACÓES DOS METODÓLOCOS DA CIENCIA ECONÓMICA

SOLANCE REGINA MARIN E RAMÓN GARCÍA FERNÁNDEZ

A EXPANSÃO DO ESCOPO TEMÁTICO DAS NEGOCIAÇÓES COLETIVAS DE TRABALHO CARLOS HENRIQUE HORN

EFEITOS DO CAPITAL SOCIAL E DO CAPITAL POLITICO NO DESENVOLVIMENTO ECONOMMICO SIMULAÇOES PARA PAISES E ESTADOS BRASILEIROS

RONALDO A. ARRAES, RICARDO CANDÉA S. BARRETO E VLADIMIR KÜHL TELES

O PROBLEMA DE RISCO MORAL NO MERCADO BRASILEIRO DE ASSISTÊNCIA MÉDICA SUPLEMENTAR LUCIANA PINTO DE ANDRADEE SABINO DA SILVA PÓRTO JÚNIOR

ANO

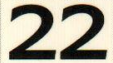

No 41

Março, 2004 
Universidade Federal do Rio Grande do Sul

Reitora: Profo. Wrana Maria Panizzi

Faculdade de Ciencias ECONOMICAS

Diretora: Prof Pedro César Dutra Fonseca

Centro de Estudos e Pesquisas Economicas

Diretor: Prof. Gentil Corazza

Departamento de Ciencias Económicas

Chefe: Prof. Ricardo Dathein

Curso de Pós-Graduação em Economia

Coordenador: Prof. Eduardo Pontual Ribeiro

Programa de Pó-Graduação em Desenvolvimento Rural

Coordenador: Prof. Jalcione Almeida

CONSElHo EDITORIAL:

Carlos G. A. Mielitz Netto (UFRGS), Eduardo A. Maldonado Filho (UFRGS), Eduardo P. Ribeiro (UFRGS), Eleutério F. S. Prado (USP), Eugênio Lagemann (UFRGS), Fernando Cardim de Carvalho (UFRJ), Fernando Ferrari Filho (UFRGS), Fernando de Holanda Barbosa (FGV/RJ), Flávio Vasconcellos Comim (UFRGS), Gentil Corazza (UFRGS), Giácomo Balbinotto Netto (UFRGS), Gustavo Franco (PUC/RJ), Jan A. Kregel (UNCTAD), João Rógério Sanson (UFSC), Joaquim Pinto de Andrade (UnB), Jorge Paulo Araújo (UFRGS), Juan H. Moldau (USP), Marcelo S. Portugal (UFRGS), Maria Alice Lahorgue (UFRGS), Paul Davidson (University of Tennessee), Paulo D. Waquil (UFRGS), Pedro C. D. Fonseca (UFRGS), Philip Arestis (Levy Economics Institut of Bard College), Roberto C. de Moraes (UFRGS), Ronald Otto Hillbrecht (UFRGS), Sabino da Silva Porto Jr. (UFRGS), Stefano Florissi (UFRGS) e Werner Baer (University of Illinois at Urbana-Champaign).

COMISSĀO EDITORIAL:

Eduardo Augusto Maldonado Filho, Fernando Ferrari Filho, Gentil Corazza, Marcelo Savino Portugal, Paulo Dabdab Waquil e Roberto Camps Moraes.

EDITOR: Prof. Fernando Ferrari Filho

Editor Adjunto: Prof. Gentil Corazza

SeCretária: Paulo Roberto Eckert

REVISÃO DE TEXTOS: Vanete Ricacheski

EdITORAÇÃo Eletrónca: Vanessa Hoffmann de Quadros

Fundador: Prof. Antônio Carlos Santos Rosa

Os materiais publicados na revista Análise Econômica são da exclusiva responsabilidade dos autores. É permitida a reprodução total ou parcial dos trabalhos, desde que seja citada a fonte. Aceita-se permuta com revistas congêneres. Aceitam-se, também, livros para divulgação, elaboração de resenhas e recensões. Toda correspondência, material para publicaçāo (vide normas na terceira capa), assinaturas e permutas devem ser dirigidos ao seguinte destinatário:

PROF. FERNANDO FERRARI FILHO Revista Análise Econômica - Av. João Pessoa, 52 CEP 90040-000 PORTO ALEGRE - RS, BRASIL Telefones: (051) 316-3513 - Fax: (051) 316-3990

Análise Econômica E-mail: rae@ufrgs.br

Ano 22, $n^{\circ} 41$, março, 2004 . Porto Alegre

Faculdade de Ciências Econômicas, UFRGS, 2004

Periodicidade semestral, março e setembro.

Tiragem: 500 exemplares

1. Teoria Econômica - Desenvolvimento Regional -

Economia Agrícola - Pesquisa Teórica e Aplicada -

Periódicos. I. Brasil

Faculdade de Ciências Econômicas,

Universidade Federal do Rio Grande do Sul. 


\title{
Necessidades e os Condicionantes da Segunda Ocupação: comentário
}

\author{
Eduardo Pontual Ribeiro*
}

\section{Introdução}

O artigo de Menezes e Carrera-Fernandez (2003) estuda o perfil e determinantes das pessoas da Região Metropolitana de Salvador (RMS) que possuem mais de uma ocupação. O tema é interessante e ainda não teve tratamento na literatura nacional. $\mathrm{O}$ artigo apresenta uma seção descritiva das características daqueles que têm mais de uma ocupação, uma seção estudando os rendimentos e uma última sobre as características mais correlacionadas com o fato de um trabalhador ter mais de uma ocupação.

O objetivo desta nota é chamar a atenção de limitações da análise que podem comprometer os resultados obtidos. A primeira está na falta de referencial teórico para o estudo. Esta falta de referencial leva à segunda limitação do texto, nas seções empíricas. As estimações apresentam problemas sérios de endogeneidade $\mathrm{e}$ deveria ser refeita. Em particular, na última seção, foi incluída uma variável explicativa no modelo Logit que é um previsor perfeito da participação, ou não, na segunda ocupação. A inclusão desta variável, além de gerar resultados numéricos espúrios, é claramente endógeno, gerando viés nas estimativas dos coeficientes.

\section{O referencial teórico}

Como dito acima, o trabalho sofre da falta de referências teóricas importantes. Implícito ao longo do texto, diante dos modelos de rendimentos e de participação empregados nas seções 3 e 4, está o modelo de maximização de utilidade com argumentos lazer e consumo usual na literatura de participação no mercado de trabalho e oferta de trabalho(veja por exemplo, Ehrenberg e Smith, 2001, cap.6). Para o caso específico de oferta de trabalho para emprego secundário, o artigo seminal sobre o tema é Shishko e Rotsker (1976). Na-

•PPGE/UFRGS e Pesquisador do CNPq. eribeiro@ufrgs.br 
quele texto, fica claro que a decisão de procurar um segundo trabalho depende de restrições na combinaçāo horas trabalhadas-rendimentos no emprego principal e os rendimentos do emprego secundário. Pode ser o caso que o salário hora no trabalho secundário seja menor ou maior que o do trabalho principal. Se não há restrições de horas no trabalho principal, necessariamente a segunda ocupação teria um rendimento-hora maior. Se, por outro lado, há restriçōes de escolha de horas na ocupação principal (que é o caso comum para assalariados), então o rendimento-hora da segunda ocupação pode ser até menor que o mesmo da ocupação principal.

Esta implicação da teoria, que identifica, de modo indireto, a necessidade da segunda ocupação não foi estudada pelos pesquisadores. As tabelas da seção 2 focam estatísticas descritivas gerais. São apresentadas informações simples (Quadro 1), que mostra que a segunda ocupação apresenta rendimentos positivos, ou inconsistentes (média de rendimentos na ocupação principal no Quadro 2 é igual à média da soma dos rendimentos da ocupação principal e adicional no Quadro 1). Da mesma forma, não causa surpresa que os rendimentos ou as horas semanais de trabalho no trabalho secundário são menores do que os do trabalho principal, pois são exatamente estas as características que definem qual é o trabalho principal e qual é o secundário. Por exemplo, o IBGE ${ }^{1}$, para pessoas com mais de um trabalho no período de referência, define como trabalho principal aquele que segue os seguintes critérios (em ordem de precedência):

" $\left.1^{\circ}\right)$ O trabalho da semana de referência no qual teve mais tempo depermanência no período de referência de 365 dias foi considerado como principal;

$2^{\circ}$ ) Em caso de igualdade no tempo de permanência no período de referência de 365 dias, considerou-se como principal o trabalho remunerado da semana de referência ao qual a pessoa normalmente dedicava maior número de horas semanais. Este mesmo critério foi adotado para definir o trabalho principal da pessoa que, na semana de referência, teve somente trabalhos não-remunerados e que apresentaram o mesmo tempo de permanência no período de referência de 365 dias; e

$\left.3^{\circ}\right)$ Em caso de igualdade, também, no número de horas trabalhadas, considerou-se como principal o trabalho da semana de referência que normalmente proporcionava maior rendimento." (IBGE, 2003, s.p.)

${ }^{1}$ Não foi possivel obter a definiçāo de trabalho secundário na PED, via internet. 
A análise do Quadro 9 tenta estudar os diferenciais de rendimentos nos trabalhos principal e secundário, mas sofre de problemas econométricos. Primeiro, ao contrário do que afirmam os autores (p.200-201), a constante não identifica um diferencial "autônomo" de rendimento. Isto porque, lembrando a fórmula de mínimos quadrados ordinários, para um modelo de regressão $y=\alpha+\beta x+\varepsilon$, e denominando a constante a e a estimativa do parâmetro $a$, temos ${ }^{2}$ $a=\bar{y}-b \bar{x}$. Desta forma, diferenças na constante podem vir de diferenças nas médias da variável dependente ou de diferenças nas explicativas (ou até nos coeficientes). Como as características pessoais são diferentes entre aqueles que tem ou não uma segunda ocupação, não é claro que diferenças de constantes levem a diferenças de salários. Segundo, as estatísticas $t$ parecem ser sobre estimadas e não robustas à heterocedasticidade. A formula empregada é $T=$ $\left(b_{\text {secund }}-b_{\text {princia }}\right) /\left(V\left(b_{\text {secund }}-b_{\text {princial }}\right)\right)^{1 / 2}$, onde $b_{\text {secund }}$ indica o coeficiente estimado no Quadro 7 e $b_{\text {princial }}$ o coeficiente estimado no Quadro 8. Podemos escrever $V\left(b_{\text {secund }}-b_{\text {princial }}\right)=V\left(b_{\text {secund }}\right)+V\left(b_{\text {princial }}\right)+\operatorname{Covar}\left(b_{\text {secund" }}\right.$ $b_{\text {princial }}$. Sendo o último termo zero, pois as pessoas das regressōes são diferentes, temos, para o coeficiente da (log) escolaridade, $T=$. $0.051 /\left((0.018)^{2}+(0.015)^{2}\right)^{1 / 2}=-2.1766$, valor muito distante do apresentado no Quadro 9. Inclusive, é possível que com a correção para heterocedasticidade não haja diferença entre os coeficientes, ao contrário do que afirmam os autores.

\section{As estimativas empíricas por análise de regressão}

A falta de referencial teórico explícito leva a problemas nas seções de análise de regressão. Na seção 3, é incluída a variável "esforço", que nada mais é do que horas trabalhadas. Esta variável é claramente endógena, dentro do modelo teórico implícito empregado pelos autores. Sua inclusão implica em viés nas estimativas. Outro problema é o viés de seleção presente no Quadro 8, ou seja, a equação de rendimentos dos trabalhadores que têm mais de uma ocupação. As pessoas que buscam uma segunda ocupação não são uma amostra aleatória da população, pois a decisão de buscar o

${ }^{2}$ Para simplificar a notação, apresento os resultados para regressão simples. A conclusão não muda no caso de regressão múltipla. 
segundo rendimento depende do salário esperado, que é a variável dependente na regressão. O viés de seleção é similar ao existente na análise de rendimentos de mulheres e sua correção é popular. Veja, por exemplo, Figueiredo Neto (1998) neste mesmo periódico.

Por fim, e mais preocupante é a inclusão da variável "log esforço do trabalho adicional", ou de modo mais transparente, horas trabalhadas na segunda ocupação, no modelo da seção 4. Esta variável é um previsor perfeito da variável dependente. Se a pessoa, não possui segunda ocupação, o valor da variável será necessariamente zero. Assim, para prever a variável dependente, o algoritmo Logit deve apenas concentrar-se nesta variável. O teste de Wald do coeficiente de horas mais de 100 vezes maior que o das outras variáveis é sintomático deste efeito. A própria referência constante na bibliografia chama a atenção para este problema:

"If one of the regressors contains a value, say $\mathrm{x}^{*}$, such that whenever this $\mathrm{x}$ is greater than $x^{*}, y$ equals 1 , and vice versa, whenever this $x$ is less than $x^{*}, y$ equals 0 , and vice versa, then the model will be a perfect predictor. (...)

For example, careless researchers sometimes include as regressors dummy variables that are identical, or nearly so, to the dependent variable. In this case, the maximization procedure will break down precisely because $\mathrm{b}^{\prime} \mathrm{x}$ is exploding during the interations. Of course this is not at all what we had in mind for a good fit" (Greene, 1997, p.892)

Desta forma, toda a inferência baseada no modelo é inválida, desautorizando as conclusōes dos autores ${ }^{3}$.

\section{Comentários Finais}

Em suma o artigo aborda um tema interessante mas problemas de falta de base teórica explicita e estimativas fortemente questionáveis exigem maiores estudos sobre o assunto.

\footnotetext{
3 Vale a pena notar que problema similar aparece em Carrera-Fernandez e Menezes (2001). Aquele artigo que estuda as características pessoais associadas à participaçāo do idoso no mercado de trabalho, usa como regressor a renda do trabalho. É claro que se a pessoa trabalha, a renda será positiva e se não participa do mercado de trabalho a renda será zero. Por outro lado, neste artigo de 2001 o modelo de participaçāo é apresentado em detalhe que seria necessário no artigo de 2003.
} 


\section{Referências Bibliográficas}

Carrera-Fernandez, J. e Menezes, W. Necessidades e os condicionantes da segunda ocupação. Análise Econômica, v.21, n.39, 189-209, 2003.

. O idoso no mercado de trabalho: uma análise a partir da Região Metropolitana de Salvador. Revista Econômica do Nordeste, v.32, 52-67, 2001.

Ehrenberg R. e Smith R. A Moderna Economia do Trabalho. São Paulo:Makron, 2001.

Figueiredo Neto, L.F. Mercado de trabalho e investimentos em capital humano. Análise Econômica,v.16, no.29, p.67-86, 1998.

Greene, W. Econometric Analysis, 3th.Ed. Upper Saddle River, NJ:Prentice Hall, 1997.

IBGE Notas técnicas, PNAD 2002. < http://www.ibge.gov.br/home/estatistica/ populacao/ trabalhoerendimento/pnad2002/notas_sintese.pdf $\geq$ acessado em 1/12/2003.

Shishko, R.e Rostker, B. The economics of multiple job holding. American Economic Review, v.66, 298-306, 1976. 